\title{
Repair of Anomalous Pulmonary Venous Connection by Catheter Intervention
}

\section{Sedigheh Saedi* and Tahereh Saedi}

Rajaei Cardiovascular Medical and Research Center, Iran University of Medical Sciences, Tehran, Iran

*Corresponding author: Sedigheh Saedi, Assistant Professor of Cardiology, Rajaei Cardiovascular Medical and Research Center, Iran University of Medical Sciences, Tehran, Iran, Tel: 009821-23921; E- mail: sedsaedi@gmail.com

Rec Date: September 22, 2017, Acc Date: October 11, 2017, Pub Date: October 18, 2017

Citation: Saedi S, Saedi T (2017) Repair of Anomalous Pulmonary Venous Connection by Catheter Intervention. Interv Cardiol J. Vol. 3 No. 3:65

\section{Abstract}

Transcatheter interventions for congenital heart anomalies are constantly improving. Although correction of anomalous pulmonary venous connection is routinely achieved through surgery, there are rare instances where the abnormal pulmonary vein has dual connections to both left atrium and the major systemic veins. Under these circumstances catheter based treatment might become a feasible option. We report a case of dual supply vertical vein connected to left upper pulmonary vein and innominate vein which was successfully obstructed by an occluder device leading to improvements in patient's condition.

Keywords: Congenital heart anomalies; Partial anomalous pulmonary venous connections

\section{Introduction}

Partial anomalous pulmonary venous connections (PAPVCs) are abnormal connections of the one or more of the pulmonary veins to the right sided heart chambers leading to left to right shunting of blood. Rarely the abnormal pulmonary vein (PV) has a dual connection to both left atrium (LA) and the superior/ inferior vena cava via a Simitar or vertical vein. Here we describe a young symptomatic man with dual drainage of an isolated PAPVC successfully repaired by catheter intervention leading to resolution of symptoms.

\section{Case Report}

A 28-year-old male presented to our cardiology clinic complaining of palpitation and dyspnea NYHA class I (The New York Heart Association Functional Classification). Palpitation was activity related and hindered his working capacity. He did not mention any past history of medical conditions or drug use. Physical examination findings were unremarkable.

Twelve lead ECG revealed sinus tachycardia with heart rate of about 110 beats/ minute, normal axis and right bundle branch block pattern without arrhythmias.
Chest X-ray findings were within normal limits. Exercise stress testing ordered to mimic patient's chief complaint of exercised induced palpitation and investigate for activity related arrhythmias was completely normal with the patient accomplishing the exercise protocol asymptomatically and achieving the functional capacity of 11 METs. Oxygen saturation in room air was $94 \%$.

Transthoracic echocardiography was performed showing normal left ventricular size and systolic function, mild right ventricular enlargement with preserved systolic function. There was no valvular abnormality and no atrial septal defects (ASD) or other intra-cardiac communications were detected. In the suprasternal view however an abnormal vessel parallel to proximal descending aorta with its color Doppler flow moving towards the transducer was detected in favor of a vertical vein raising the likelihood of an isolated left sided partial anomalous pulmonary venous drainage/connection (PAPVC) (Figure 1).

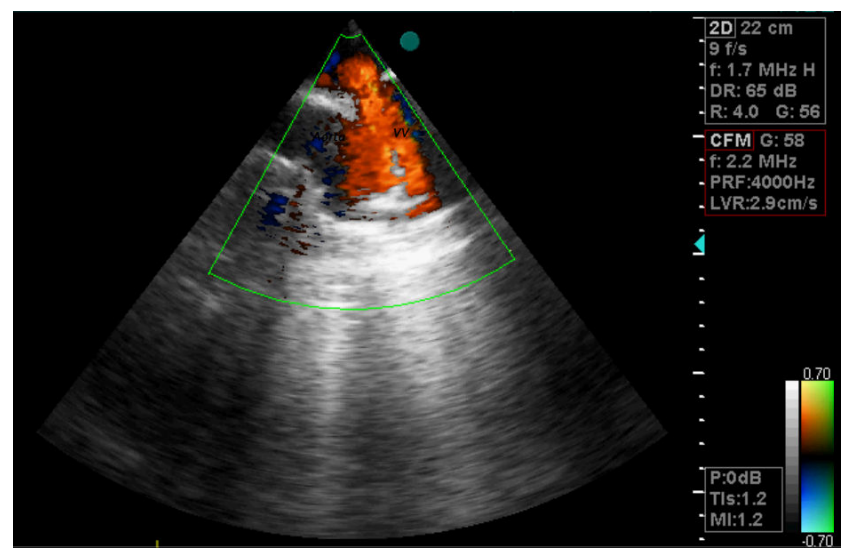

Figure 1 Suprasternal echocardiographic view demonstrating left vertical vein(VV) with direction of flow towards transducer.

In transoesophageal echocardiography the left upper pulmonary vein was seen to be attached to the left atrium, but it also seemed to drain to a vertical vein. No ASD or patent foramen ovale was present. To further investigate this unusual anatomy, cardiac magnetic resonance imaging was performed and revealed the abnormal pulmonary vein had a dual connection both to left atrium and vertical vein (hence indirectly to the right atrium) (Figure 2). 


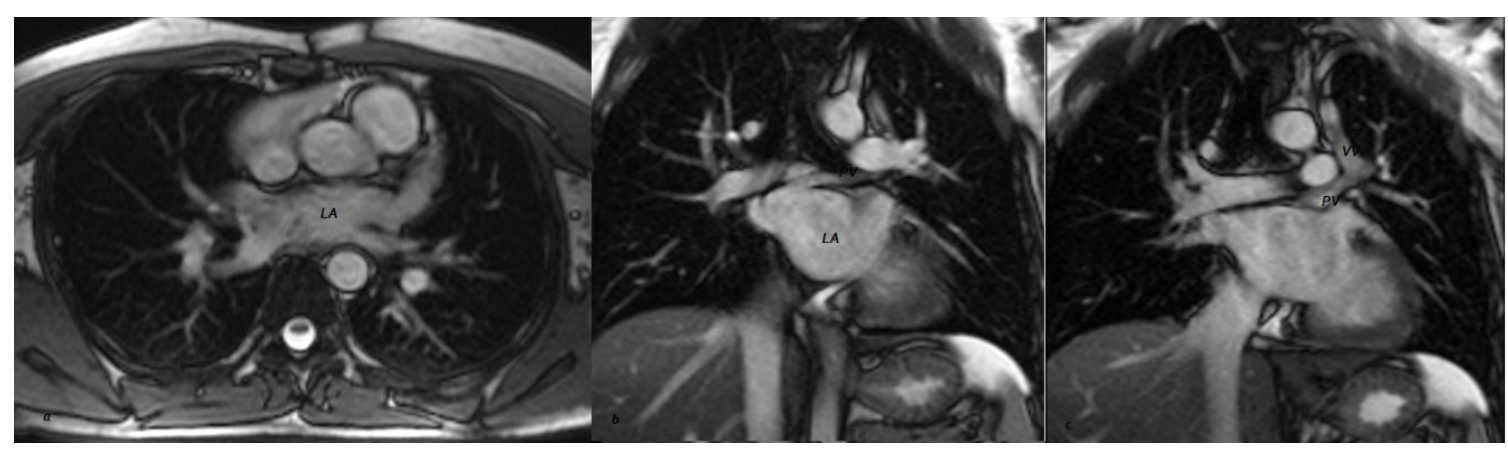

Figure 2 Cardiac magnetic resonance images, a) transverse image showing connection of all four PVs to LA, b and c) Sequential images showing connection of left upper PV to LA and vertical vein(VV).

No other associated abnormality was reported. The patient underwent cardiac catheterization that confirmed the connection of the vertical vein to both innominate vein and the left atrium via the left upper pulmonary vein (Figures 3 and 4).

The systolic pulmonary arterial pressure was $32 \mathrm{mmHg}$. As the RV had become enlarged and the patient was symptomatic, it was decided to occlude the vertical vein percutaneously.

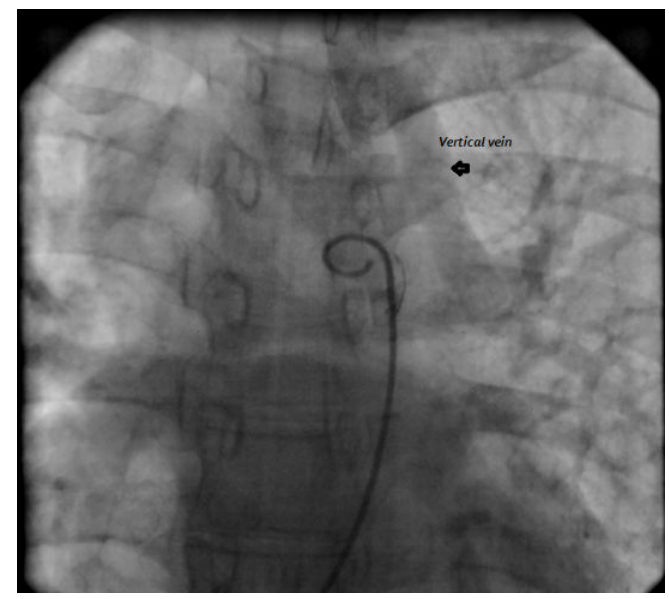

Figure 3 Pulmonary artery injection in showing vertical vein in levo-phase.

A 0.035 guide wire and a multipurpose catheter was passed from the femoral vein through inferior vena cava(IVC), RA, SVC, innominate vein, vertical vein and the left upper pulmonary vein. Then the guide wire was exchanged for an extra-stiff guide wire over which a long 12-F delivery sheath was placed.

To achieve complete obstruction with minimal risk for residual shunting a $12 \mathrm{~mm}$ symmetric muscular ventricular septal occluder device (Cera occluder, Lifetech Scientific, China) was chosen, loaded on the cable and advanced in the delivery sheath.

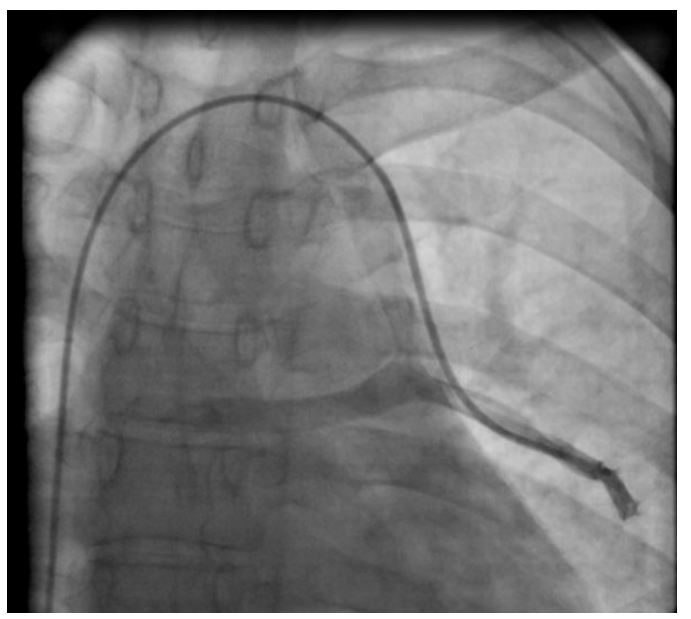

Figure 4 Contrast injection in vertical vein showing connection of left upper pulmonary vein to left atrium.

Device size was chosen based on CMR and angiographic measurements. After confirming the appropriate position, the device was released at the end of the vertical vein. Control contrast injection and levo-phase imaging confirmed successful positioning of the device with no residual leakage (Figure 5).

Follow up echocardiography 24 hours after the procedure showed eliminated flow of the vertical vein and proper position of the device with no compressive effect on adjacent structures and no clot.

The patient was discharged on aspirin $80 \mathrm{mg}$ and clopidogrel $75 \mathrm{mg}$ daily. In one-month clinic visit the patient stated that the palpitation had disappeared and functional capacity improved. Transthoracic echocardiography one, six and twelve months post- procedure confirmed satisfactory results with reduction in right ventricle end diastolic diameter and good device position. 


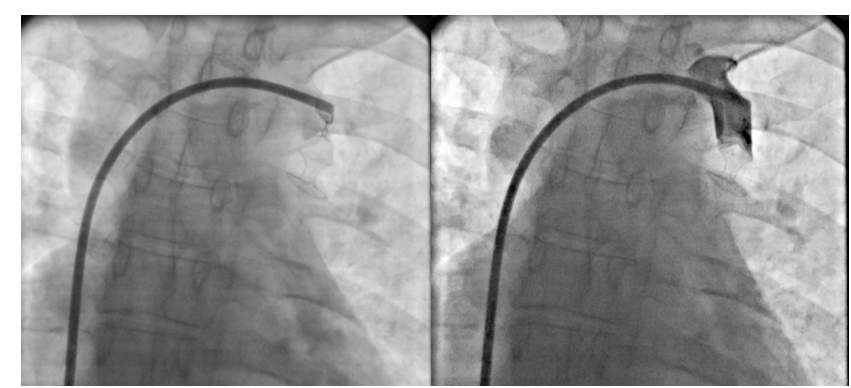

Figure 5 VSD occluder device positioned in the duct with contrast injection showing no residue.

\section{Discussion}

Partial anomalous pulmonary venous connections have different anatomic sub-types with all resulting in left to right shunt, volume overload and enlargement of the right sided cardiac chambers. Therefore, whenever patients with unexplained right ventricular enlargement are encountered in clinical practice or during echocardiographic evaluation, vigorous search for PAPVCs should be performed. Right sided anomalous pulmonary veins usually have abnormal connections to SVC, RA, coronary sinus or drain to IVC via a Scimitar vein. Left sided PAPVCs generally connect to right side chambers via a vertical vein with flow towards innominate vein, SVC and RA [1].

PAPVCs are frequently associated with ASDs. In the majority of cases the ASD is of sinus venosus type which is believed to be due to embryonic developmental failure of the wall between the sinus venosus and the pulmonary veins $[1,2]$. As a rule, affected patients with significant left to right shunting are referred for surgical correction. However isolated dual supply vertical vein draining the left upper pulmonary vein are rarely present in these patient population and must be kept in mind while assessing PAPVCs; as the anomaly could be amenable to catheter intervention thus obviating the need for surgery.

There are few reported cases of percutaneous obstruction of abnormal PAPVC flow in the literature. These cases included patients with dual drainage of pulmonary veins to IVC and left atrium via a Scimitar Vein or to SVC and LA via a vertical vein. Occlusion was accomplished by using coil or Amplatzer vascular plugs [3-6]. We used a symmetric VSD occluder in our patient that based on the anatomy seemed to achieve complete occlusion. However no follow up study on this type of intervention exists. There is also no evidence on the risks of dislodgment, clot formation, thromboembolism and the need for antiplatelet drugs. We decided to administer dual antiplatelet therapy to our patient for a period of three to six months similar to transcatheter ASD/VSD closure procedures.

\section{Conclusion}

In conclusion, careful and comprehensive evaluation of every individual with congenital heart disease is necessary to determine the optimal management strategy. For PAPVCs through delineation of anatomy may lead to successful treatment by transcatheter methods in selected cases rather than a routine surgical approach.

\section{References}

1. Wilson W, Horlick E, Benson L (2015) Successful transcatheter occlusion of an anomalous pulmonary vein with dual drainage to the left atrium. Catheter Cardio Inte 85: 1212-1216.

2. Saedi S, Salehi A, Saedi T, Hashemi MJ (2013) Association of left ventricular diverticula and sinus venosus atrial septal defect. Congenit Heart Dis 8: E145-E148.

3. Forbess LW, O'Laughlin MP, Harrison JK (1998) Partial anomalous pulmonary venous connection. Demonstration of dual drainage allowing non-surgical correction. Catheter Cardiovasc Diagn 44: 330-335.

4. Kasarala G, Fiore A, Schowengerdt K, Jureidini S (2011) Partial anomalous pulmonary venous return: Transcatheter repair. Paediatr Cardiol 32: 1238-1240.

5. Baspinar O, Irdem A, Kiline M (2013) Transcatheter repair of partial anomalous pulmonary venous drainage using an Amplatzer cardiac plug in a postoperative patient with Tetralogy of Fallot. Pediatr Cardiol 34: 1043-1046.

6. Singh $H$, Luthra $M$, Bharadwaj $P$, Kumar R (2007) Interventional rerouting of scimitar vein to left atrium using an Amplatzer vascular plug. Congenit Heart Dis 2(4):265-9. 